\title{
Validation of diffusion spectrum magnetic resonance imaging with manganese-enhanced rat optic tracts and ex vivo phantoms
}

\author{
Ching-Po Lin, ${ }^{\text {a }}$ Van Jay Wedeen, ${ }^{\mathrm{b}}$ Jyh-Horng Chen, ${ }^{\mathrm{a}, *}$ Ching Yao, ${ }^{\mathrm{c}}$ \\ and Wen-Yih Isaac Tseng ${ }^{\mathrm{d}, *}$ \\ ${ }^{a}$ Interdisciplinary MRI/MRS Lab, Department of Electrical Engineering and Institute of Biomedical Engineering, \\ National Taiwan University, Taipei, Taiwan \\ ${ }^{\mathrm{b}}$ Department of Radiology, NMR Center, Massachusetts General Hospital, Boston, MA, USA \\ ${ }^{\mathrm{c}}$ Division of Medical Engineering Research, National Health Research Institute, Taipei, Taiwan \\ ${ }^{\mathrm{d}}$ Center for Optoelectronic Biomedicine, National Taiwan University College of Medicine and Department of Radiology, National Taiwan University \\ Hospital, National Taiwan University College of Medicine, Taipei, Taiwan
}

Received 7 October 2002; revised 12 February 2003; accepted 3 March 2003

\begin{abstract}
Diffusion spectrum imaging (DSI) has been demonstrated to resolve crossing axonal fibers by mapping the probability density function of water molecules diffusion at each voxel. However, the accuracy of DSI in defining individual fiber orientation and the validity of Fourier relation under finite gradient pulse widths are not assessed yet. We developed an ex vivo and an in vivo model to evaluate the error of DSI with gradient pulse widths being relatively short and long, respectively. The ex vivo model was a phantom comprising sheets of parallel capillaries filled with water. Sheets were stacked on each other with capillaries crossed at $45^{\circ}$ or $90^{\circ}$. High-resolution T2-weighted images (T2WI) of the phantom served as a reference for the orientation of intersecting capillaries. In the in vivo model, manganese ions were infused into rats' optic tracts. The optic tracts were enhanced on T1-weighted images (T1WI) and served as a reference for the tract orientation. By comparing DSI with T2WI, the deviation angles between the primary orientation of diffusion spectrum and the $90^{\circ}$ and $45^{\circ}$ phantoms were $1.19^{\circ} \pm 4.82^{\circ}$ and $-0.71^{\circ} \pm 4.91^{\circ}$, respectively. By comparing DSI with the T1WI of rat optic tracts, the deviation angle between primary orientation of diffusion spectrum and optic tracts was $-0.41^{\circ} \pm 6.18^{\circ}$. In addition, two sequences of DSI using short and long gradient pulses were performed in a rat brain. The bias of the primary orientation between these two sequences was approximately $10^{\circ}$. In conclusion, DSI can resolve crossing fiber orientation accurately. The effect of finite gradient pulse widths on the primary orientation is not critical. (C) 2003 Elsevier Science (USA). All rights reserved.
\end{abstract}

Keywords: Diffusion spectrum; Validation; $\mathrm{Mn}^{2+}$; MRI

\section{Introduction}

Water molecule displacement restricted in an organized material reflects geometrical properties of the underlying microenvironment. Based on this tenet, diffusion nuclear magnetic resonance (NMR) has long been exploited to investigate microstructures (Mansfield and Grannell, 1973; Grannell and Mansfield, 1975). One approach of diffusion

\footnotetext{
* Corresponding author. Fax: + 886-2-2369-9465 (J.-H. Chen), + 8862-2392-6922 (W.-Y.I. Tseng).

E-mail addresses: chen@me.ee.ntu.edu.tw (J.-H. Chen), wytseng@ ha.mc.ntu.edu.tw (W.-Y.I. Tseng).
}

NMR experiments is $\mathbf{q}$-space imaging. It samples multiple diffusion-attenuated images with different spatial modulations of magnetization generated by diffusion-sensitive gradients (Callaghan et al., 1988; Cory and Garroway, 1990; Callaghan, 1991; Callaghan et al., 1991). In the limit where the diffusion-sensitive gradient approximates a delta function, the signal sampled in the space of spatial modulation of magnetization, dubbed $\mathbf{q}$ space, relates to spatial probability of translational displacement of water molecules by Fourier transform (FT) (Stejskal, 1965). The width of the translational displacement distribution has been shown to reflect the dimensions of the microstructures, demonstrate the existence of restriction, and characterize water mobility in 

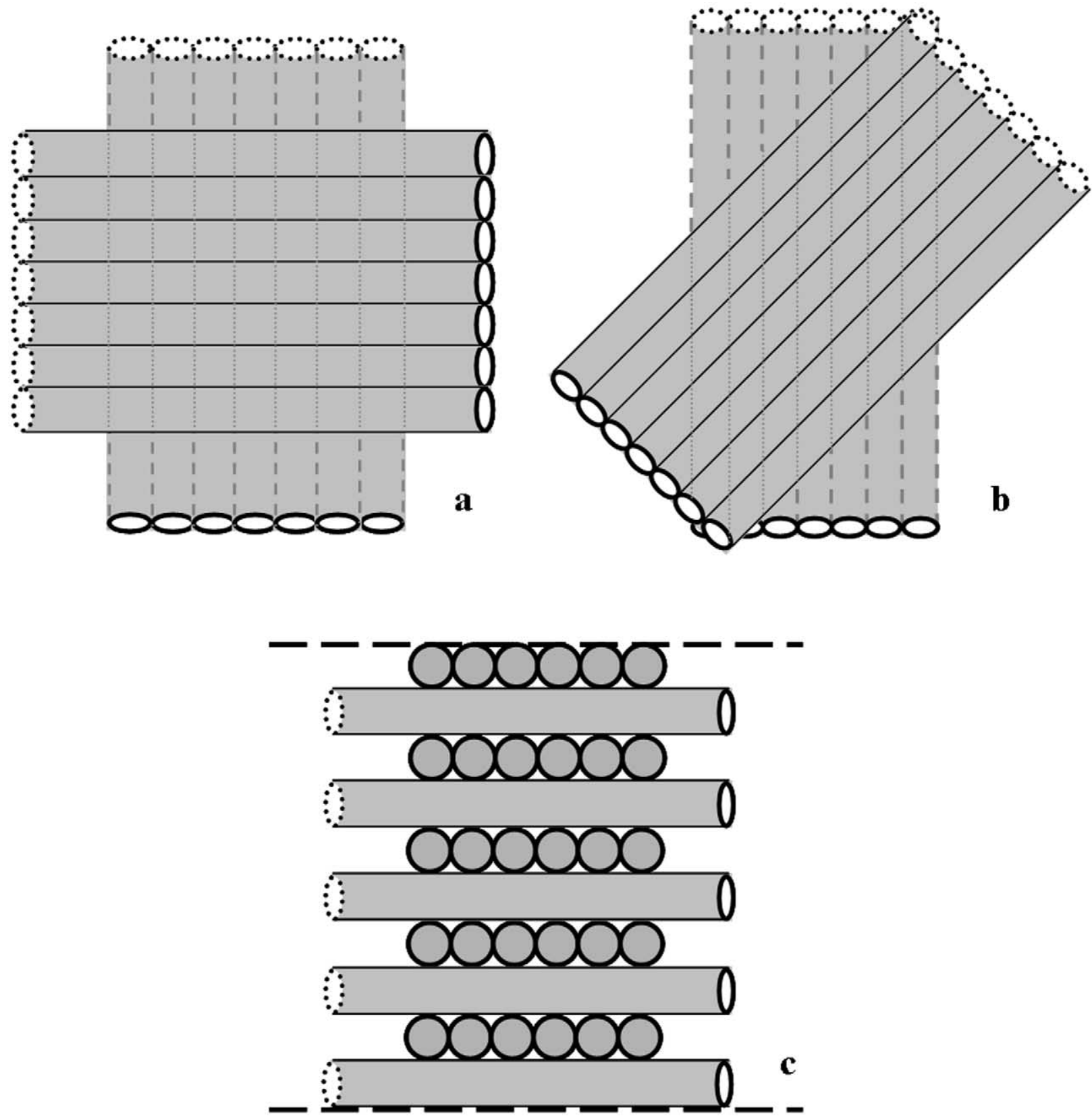

Fig. 1. Design of capillary phantoms. Capillaries were filled with water and sheets of two different orientation, $90^{\circ}$ (a) and $45^{\circ}$ (b), were stacked on each other in an interleaved fashion (c).

distinct anatomical compartments. (King et al., 1994; Hills et al., 1996; Assaf and Cohen, 1999; Assaf et al., 2000; Kuchel et al., 2000). Q-space imaging has been widely used to measure one-dimensional length scales in predetermined directions. Extension of this method to the mapping of three-dimensional (3D) displacement probability requires sampling over the 3D q space. Though theoretically feasible for such study, extraordinarily long acquisition time is required to cover a wide range of $\mathbf{q}$ space.

Another approach is diffusion tensor imaging (DTI). Instead of measuring dimensions, it detects orientation of the microstructure by approximating 3D translational displacement distribution with a diffusion tensor (Basser et al., 1994). The orientation of an anisotropic tissue can be determined from the principal orientation of the diffusion tensor at each pixel location. Being able to determine axonal fiber directions at pixel-by-pixel basis, DTI has demonstrated its potential in mapping neural fiber tracts (Conturo et al., 1999; Mori et al., 1999; Basser et al., 2000; Stieltjes et al., 2001; Gossl et al., 2002; Parker et al., 2002). Despite its clinical relevance, DTI faces a challenge in resolving individual fiber orientation within a voxel where more than 

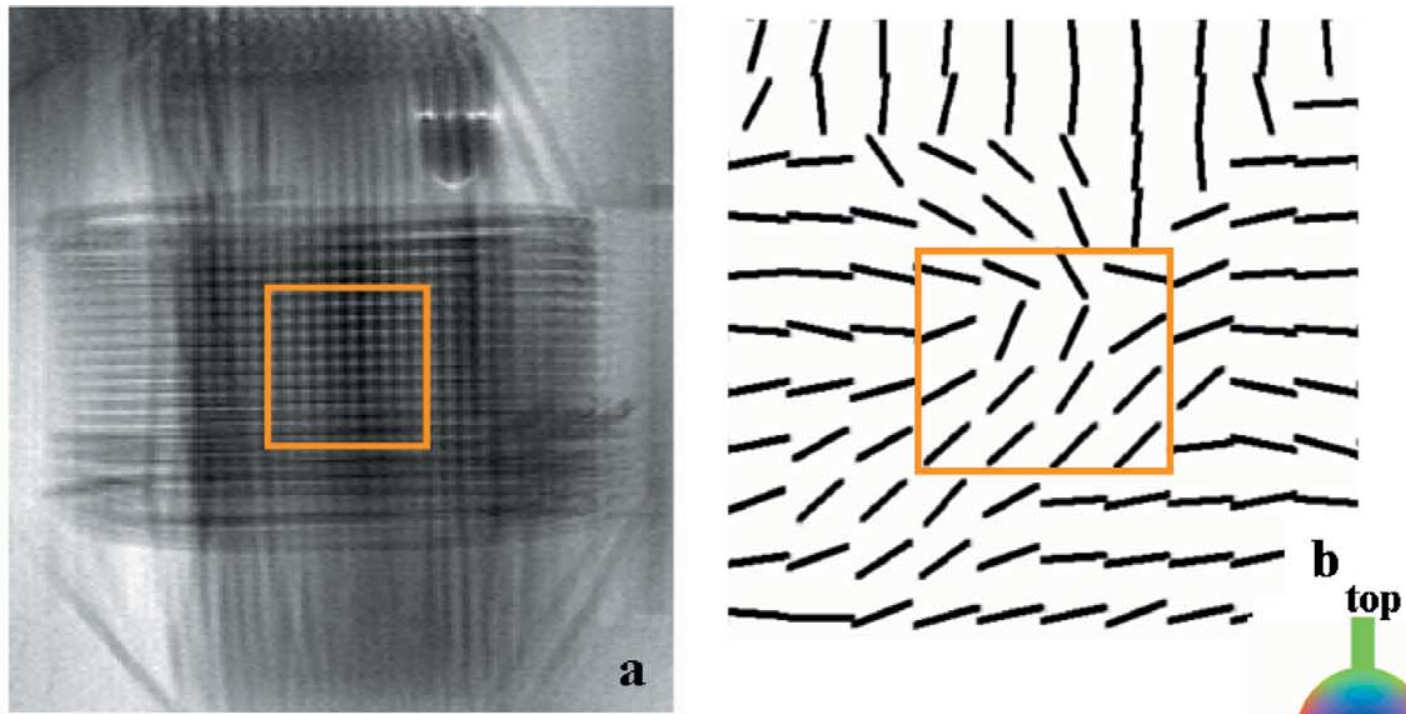

$\mathbf{a}$
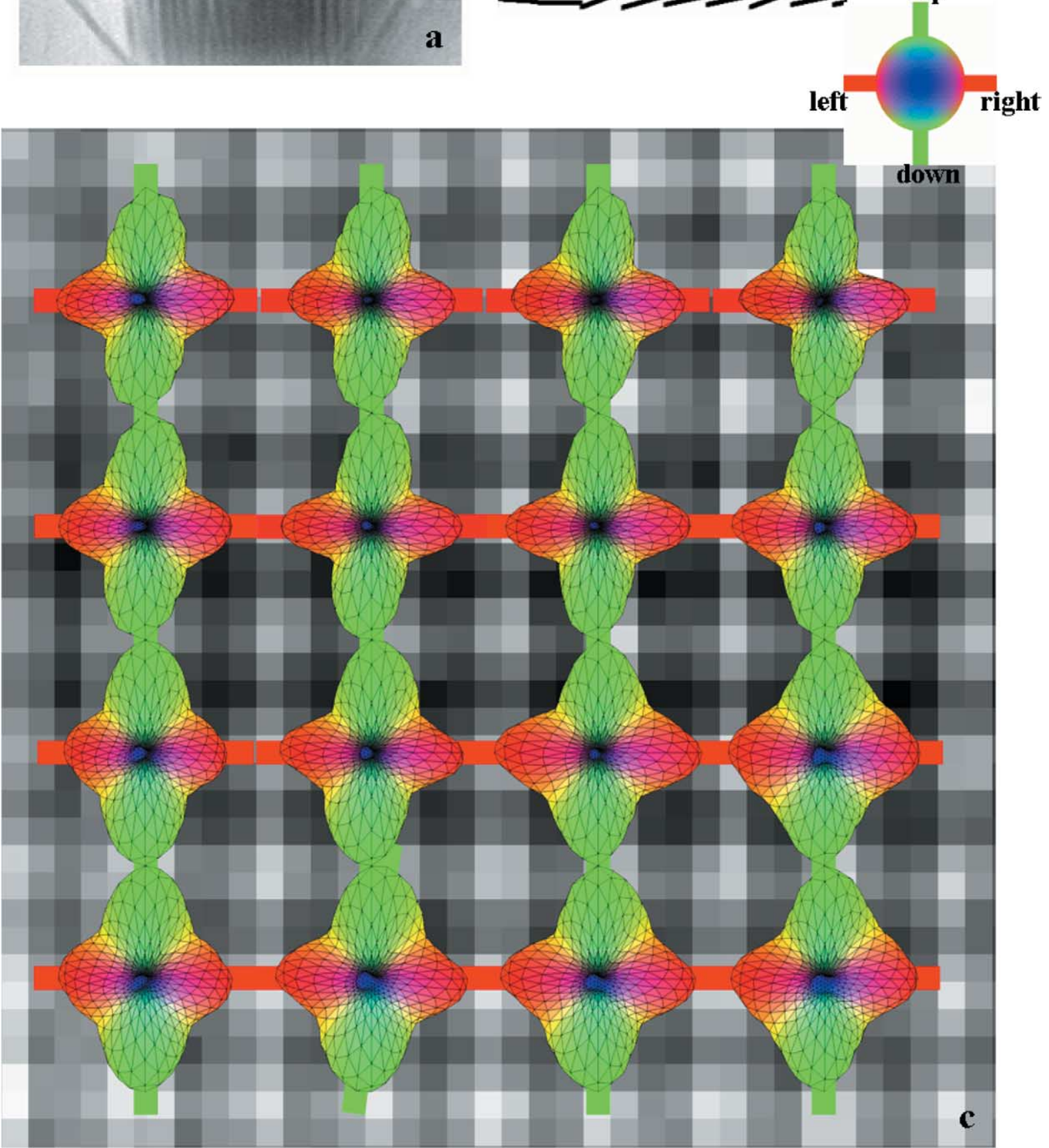

Fig. 2. The capillary phantom in $90^{\circ}$ intersection between sheets. High-resolution T2WI served as a reference to evaluate the error of DSI (a). The image in (b) is the mapping of the first eigenvector of the diffusion tensor, and the image in (c) is the mapping of ODF and its primary orientation. The region of interest is enclosed by a rectangle in (a). We can see that ODF defines the intersecting capillaries orientation correctly (c), but the first eigenvector of the diffusion tensor fails to show correct orientation for either one of the capillaries (b). The rectangles enclose the same ROI as that in (c). The orientation of ODF, and the whisker orientation in the following figures, is color-coded according to the scheme indicated in the insert. 

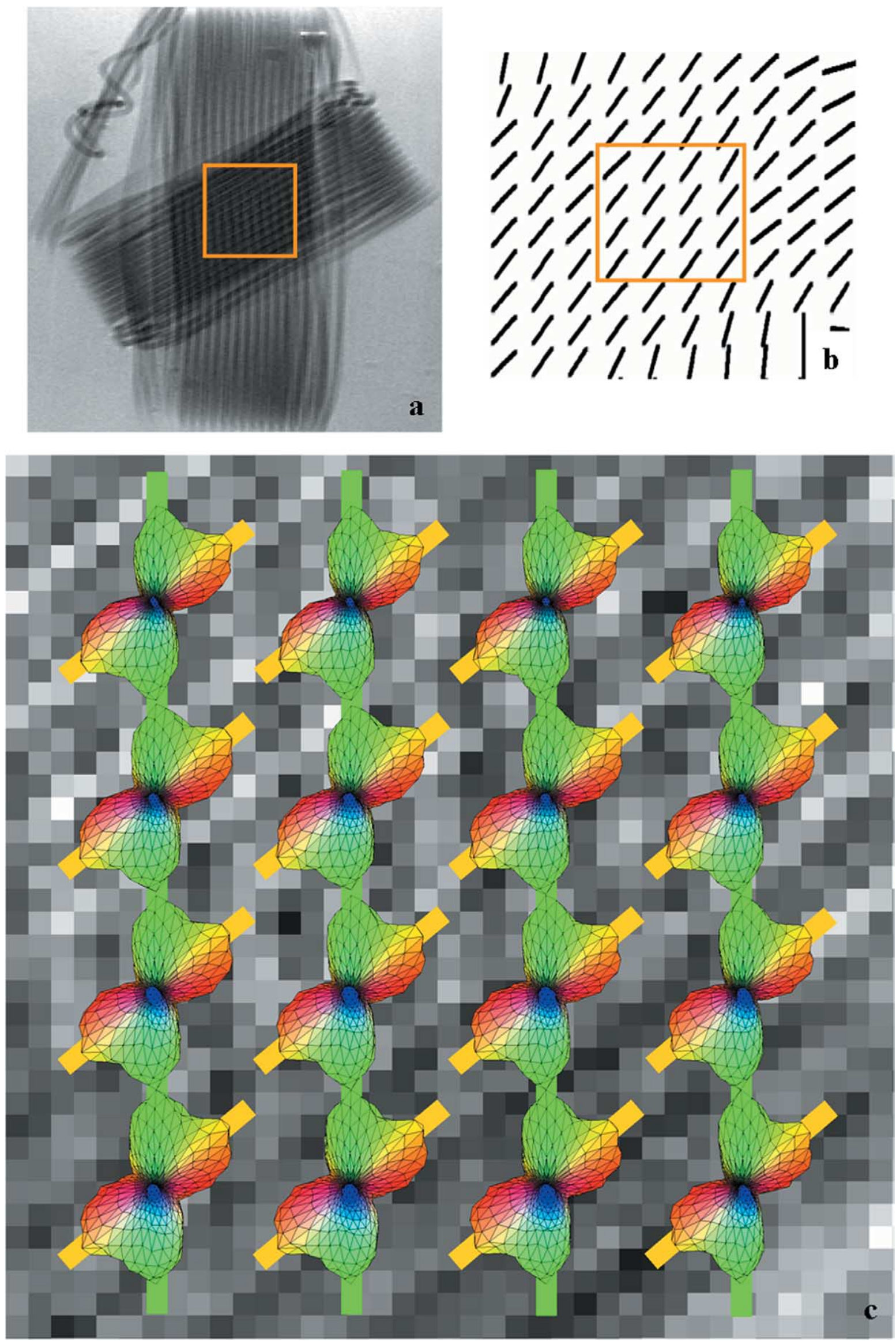

Fig. 3. The capillary phantom in $45^{\circ}$ intersection between sheets. High-resolution T2WI of the phantom served as a reference for the orientation of intersecting capillaries (a). The first eigenvector of the diffusion tensor is erroneous in defining the orientation of intersecting capillaries (b), but ODF determines the orientation correctly (c). The rectangles enclose the same ROI as that in (c). 
one fiber tract cross each other. As illustrated by Wiegell et al. (2000), in voxels containing crossing fibers the directions of the first and the second eigenvectors of the diffusion tensor became ambiguous. To address this problem, Tuch et al. (1999) and Frank (2001, 2002) sampled diffusionweighted images of the brain by applying diffusion gradients in icosahedral tessellations of a sphere. Reconstruction of 3D distribution of diffusion coefficients showed multipolar patterns in regions of fiber crossing in contrast to bipolar ones in regions of coherent fibers. The diffusion sensitivity, i.e., the $b$ value, they used in their experiments was around 2000 to $3000 \mathrm{~s} \mathrm{~mm}^{-2}$, close to those used in clinical scanners. $B$ values used in clinical regime probe water molecular displacement on a coarse length scale (Basser, 2002) and are probably too low to resolve complex fiber crossing.

To determine the directions of complex crossing fibers within a voxel, Wedeen et al. (2000) proposed a technique, called diffusion spectrum imaging (DSI), to sample diffusion-attenuated images systematically over the 3D q space. More than 500 data were acquired with $\mathbf{q}$ corresponding to Cartesian grids within a sphere where the grid spacing $|\mathbf{q}|=$ 70 radian $\mathrm{mm}^{-1}$ and the maximum $b$ value $\left(b_{\max }\right)=20,000$

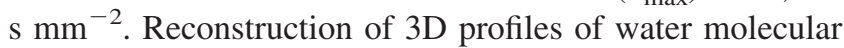
displacement (or probability density function, PDF) was obtained by 3D FT of the sampled signal $E(\mathbf{q})$ in $\mathbf{q}$ space (Callaghan, 1991). The profiles in periventricular white matter demonstrated three-way crossing from coronal radiata, longitudinal fasciculus, and corpus callosum. Though the results were consistent with known histology, the accuracy of DSI is yet to be validated. Another more fundamental question of this study is that it was performed in a clinical scanner with the maximum gradient strength of 40 $\mathrm{mT} / \mathrm{m}$. The gradient duration had to be turned on for about $64 \mathrm{~ms}$ given diffusion time $\Delta=66 \mathrm{~ms}$. In this case, the majority of translational motion of water molecules occurred during the gradient duration $\delta$, and $\mathbf{q}$ was still evolving during most of the measurement. The validity of Fourier relation between $E(\mathbf{q})$ and PDF is questionable because the essential requirement for this relation, that is, $\delta \ll \Delta$, is not satisfied (Stejskal, 1965). The effects of finite-gradient pulse widths on the measured signal $E(\mathbf{q})$ with respect to the dimensions of restrictive environment have been studied with computer simulations or analytical approximations (Balinov et al., 1993; Blees, 1994; Mitra and Halperin, 1995). It is found that the dimension of the measured object is underestimated with an increase in the width of the gradient pulse. Although the measurement under finite gradient pulse widths appears smaller than the actual size, it is not clear whether this bias will affect the orientation of the local maxima in 3D PDF.

Therefore, the purpose of this article was, first, to quantify the accuracy of DSI that met the requirement $\delta \ll \Delta$ and, second, to assess the accuracy of DSI that did not meet the requirement. We developed two models, capillary phan- toms for the first experiment, and manganese-enhanced rat optic tracts for the second experiment. The capillary phantoms consisted of sheets of parallel capillaries filled with water. To simulate fiber crossing, sheets were stacked on each other with capillaries of neighboring sheets crossed in $45^{\circ}$ or $90^{\circ}$. We used high-resolution T2-weighted images (T2WI) as a reference to determine the orientation of the intersecting capillaries. By comparing DSI with T2WI, we quantified the angular deviation between the orientation of the PDF and that of the capillaries. In the rat model, we infused manganese ions into the vitreous body of rats' eyeballs. The optic tracts were visualized by contrast enhancement effect on T1-weighted images (T1WI). The rat model was previously used to validate the feasibility of defining the axonal fiber orientation by DTI (Lin et al., 2001). Here we used the same model to evaluate the errors of DSI in defining fiber orientation in vivo. For comparison, we also assessed the accuracy of DTI with the same models. Finally, the deviation of the primary orientation of diffusion spectrum obtained from two diffusion sequences, one with short and another with long gradient pulse widths, was compared to estimate the effect of gradient pulse widths on the primary orientation of diffusion spectrum.

\section{Materials and methods}

\section{Phantom model}

We design a phantom model to simulate intersecting fibers. It consisted of sheets of parallel plastic capillaries with the inner diameter (i.d.) of $50 \mu \mathrm{m}$ and outer diameter (o.d.) of $350 \mu \mathrm{m}$ (PTFE ultramicrobore tubing P-06417-70, Cole-Parmer Instrument, Vernon Hills, IL). As shown in Fig. 1, the capillaries were filled with water, and sheets of two different orientation were stacked on each other in an interleaved fashion. To secure the orientation, we wounded the plastic capillaries and fastened them to a firm plastic plate notched at the corners. Two phantoms, one with capillaries intersecting at $45^{\circ}$ and the other at $90^{\circ}$, were built.

We performed MRI experiments in a 3T Medspec/Biospec MRI system (Bruker Companies, Ettlingen, Germany) with an inserted microgradient system, i.d. $=6 \mathrm{~cm}$ and the maximal gradient intensity $=1000 \mathrm{mT} \mathrm{m}^{-1}$. A microquadrature volume coil with i.d. of $3.5 \mathrm{~cm}$ was used for RF transmission and reception. T2WI and DSI were acquired with the same field of view (FOV), $22 \mathrm{~mm}$, and slice thickness $3.5 \mathrm{~mm}$. We used a RARE (rapid acquisition with relaxation enhancement) sequence to obtain T2WI, TR/TE $=3500 / 58.5 \mathrm{~ms}$ with matrix size $=256^{2}$, yielding in-plane resolution of $0.09 \mathrm{~mm}$. Images of DSI were acquired with a stimulated echo-pulsed gradient sequence, TR/TE $=2000 /$ $19.5 \mathrm{~ms}$ and matrix size $=32^{2}$, yielding in-plane resolution of $0.72 \mathrm{~mm}$. The diffusion-encoding scheme constituted 515 
diffusion-encoding directions, each corresponding to a point in the space of spatial modulation q. These points were located at isotropic 3D grid points within a spherical volume, the radius of which was equal to five times that of the grid width. The spatial modulation $\mathbf{q}$ is defined by $\mathbf{q}=$ $\gamma \mathbf{g} \delta / 2 \pi$, where $\gamma$ is the gyromagnetic ratio, $\mathbf{g}$ is the diffusion-encoding gradient, and $\delta$ is the time duration of the diffusion-encoding gradient. While keeping the gradient duration, $\delta$, equal to $6 \mathrm{~ms}$ and diffusion time, $\Delta$, equal to $250 \mathrm{~ms}$, DSI was encoded by varying the gradient magnitude, $|\mathbf{g}|$, from 0 to $112 \mathrm{mT} \mathrm{m}^{-1}$ yielding diffusion sensitivity $\mathrm{b}$ from $0 \mathrm{~s} \mathrm{~mm}^{-2}$ to $b_{\max }=8,000 \mathrm{~s} \mathrm{~mm}^{-2}$. The scan time for the DSI experiment was about $9 \mathrm{~h}$.

\section{Rat model}

All the procedures of animal experiment adhered to the Guidelines for Care and Use of Experimental Animals of the Laboratory Animal Center in National Taiwan University. The details of procedures were described previously (Lin et al., 2001). Briefly, three adult Wistar rats were anesthetized by intraperitoneal injection of sodium pentobarbital at a dose of $0.05 \mathrm{mg}$ per gram of body weight. We infused manganese chloride solution, $0.8 \mathrm{~mol} / \mathrm{L}$ in concentration and $2 \mu \mathrm{l}$ in amount, into the vitreal cavity of rats' eyes with a micropipette needle. Ten hours after the infusion, rats were euthanized under deep general anesthesia with intraperitoneal injection of $100 \mathrm{mg} / \mathrm{kg}$ sodium pentobarbital and placed in prone position in an acrylic semicylindrical holder with the head fixed by foam pads. The holder was then put into a mini quadrature coil for scanning. We acquired sagittal T1WI and T2WI as localizers and from those determined two oblique slices with different orientation. One slice contained optic tracts from the bilateral retina to the optic chiasm, while the other slice covered area from the optic chiasm to the superior colliculus (SC). In this way, we obtained optic tracts from retina to $\mathrm{SC}$ in two single-slice planes.

A gradient system with the i.d. of $12 \mathrm{~cm}$ and the maximal gradient intensity of $200 \mathrm{mT} \mathrm{m}^{-1}$ was inserted into the $3 \mathrm{~T}$ magnet for the rat study. A mini quadrature volume coil with the i.d. of $6 \mathrm{~cm}$ was used for RF transmission and reception. Both T1WI and DSI had the same FOV, $40 \mathrm{~mm}$, and slice thickness, $1.2 \mathrm{~mm}$. An inversion recovery gradient echo sequence was performed to obtain T1WI. The flip angle was $75^{\circ}$, TR/TE/TI $=505 / 5.1 / 320 \mathrm{~ms}$, and matrix size $=256^{2}$, yielding in-plane resolution of $0.15 \mathrm{~mm}$. DSI was performed using a spin echo pulsed gradient sequence with the same DSI encoding scheme as the phantom model. $\mathrm{TR} / \mathrm{TE}=2000 / 51.5 \mathrm{~ms}$ and matrix size $=128^{2}$, yielding in-plane resolution of $0.3 \mathrm{~mm}$. With $\delta=17 \mathrm{~ms}, \Delta=25 \mathrm{~ms}$, and diffusion gradients varying from 0 to $180 \mathrm{mTm}^{-1}$, we obtained diffusion attenuated images with $b$ values changing from 0 to $13,000 \mathrm{~s} \mathrm{~mm}^{-2}$. The DSI experiment lasted for about $36 \mathrm{~h}$.
Primary orientation of diffusion spectrum with $\delta \sim \Delta$ compared with DSI with $\delta \ll \Delta$

One adult Wistar rat was euthanized under deep general anesthesia with intraperitoneal injection of $100 \mathrm{mg} / \mathrm{kg}$ sodium pentobarbital. After being exsanguinated, the rat was perfused transcardially with physiological saline followed by $4 \%$ formaldehyde solution. Rat brain was dissected from the cranium and was placed in an acrylic holder filled with $4 \%$ formaldehyde solution.

MR experiment was performed with the same microgradient system as that used in the phantom models. To compare the primary orientation of diffusion spectrum between the conditions of $\delta \sim \Delta$ and $\delta \ll \Delta$, two DSI data sets were acquired using a spin echo sequence and a stimulated echo sequence, respectively, with identical FOV $(=20 \mathrm{~mm})$ and spatial resolution $\left(=0.3 \times 0.3 \times 1 \mathrm{~mm}^{3}\right)$. The spin echo pulsed gradient sequence used TR/TE $=1000 / 23.4 \mathrm{~ms}$, number of excitation $(\mathrm{NEX})=2$, and $\delta / \Delta=7 / 12.3 \mathrm{~ms}$. The stimulated echo pulsed gradient sequence used TR/TE $=$ $1000 / 15.6 \mathrm{~ms}$, NEX $=7$, and $\delta / \Delta=3.1 / 50 \mathrm{~ms}$. With diffusion gradients varying from 0 to $900 \mathrm{mT} \mathrm{m}^{-1}$, we obtained 515 diffusion-attenuated images with $b$ values changing from 0 to $28,000 \mathrm{~s} \mathrm{~mm}^{-2}$.

Images of DSI obtained from spin echo and stimulated echo sequences were coregistered, and pixel-by-pixel comparison of the primary orientation of diffusion spectrum was made throughout the whole imaged brain.

\section{Diffusion spectrum and diffusion tensor reconstruction}

Diffusion spectrum analysis is based on the relationship that echo signal $E(\mathbf{q})$ and diffusion PDF $P_{\mathrm{s}}(\mathbf{R})$, or the average propagator, is a FT pair (Callaghan, 1991; Wedeen et al. 2000), i.e.,

$$
E(\mathbf{q})=\int P_{s}(\mathbf{R}, \Delta) \exp [i 2 \pi \mathbf{q} \mathbf{R}] d \mathbf{R}=F\left\{P_{s}(\mathbf{R})\right\}
$$

where $\mathbf{R}$ is the relative displacement of water molecules. Using Eq. (1), we obtained 3D PDF by 3D FT of the diffusion-attenuated signals, $E(\mathbf{q})$.

To visualize the $3 \mathrm{D}$ PDF while preserving the salient orientation information we projected the 3D PDF onto a sphere by integrating over the radial dimension. The radial projection of the diffusion spectrum, called fiber orientation distribution function (ODF), $\psi(\mathbf{u})$, represents the probability of finding a fiber oriented in the direction of the unit vector, $\mathbf{u}$.

$$
\psi(\mathbf{u})=\int_{0}^{r_{\max }} P_{s}(r \mathbf{u}) r^{2} d r
$$

We determined the primary orientation of PDF, $\mathbf{d}_{1}$, by selecting the orientation of ODF corresponding to greatest $\psi$. To enhance the visualization, we color coded the orien- 

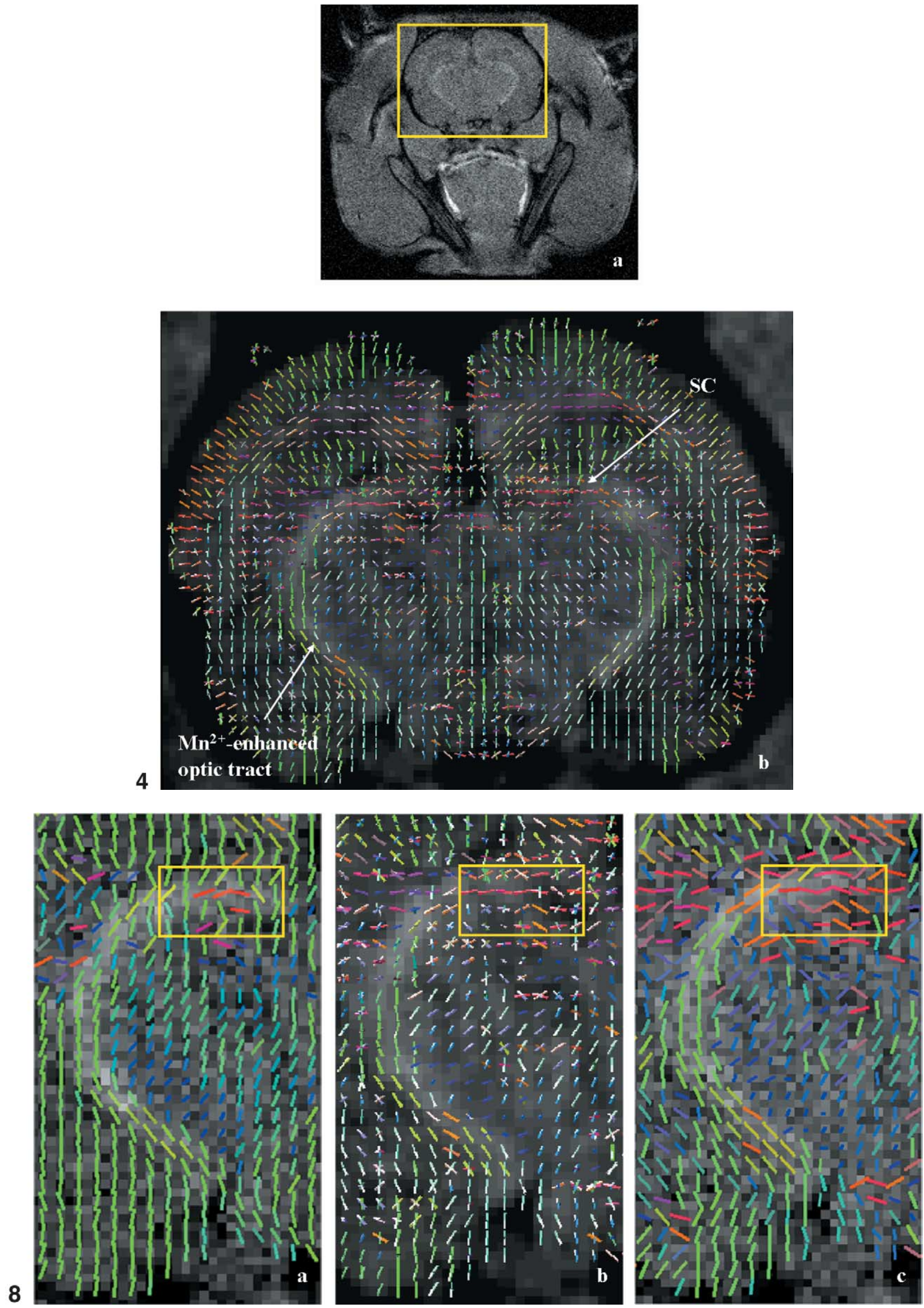


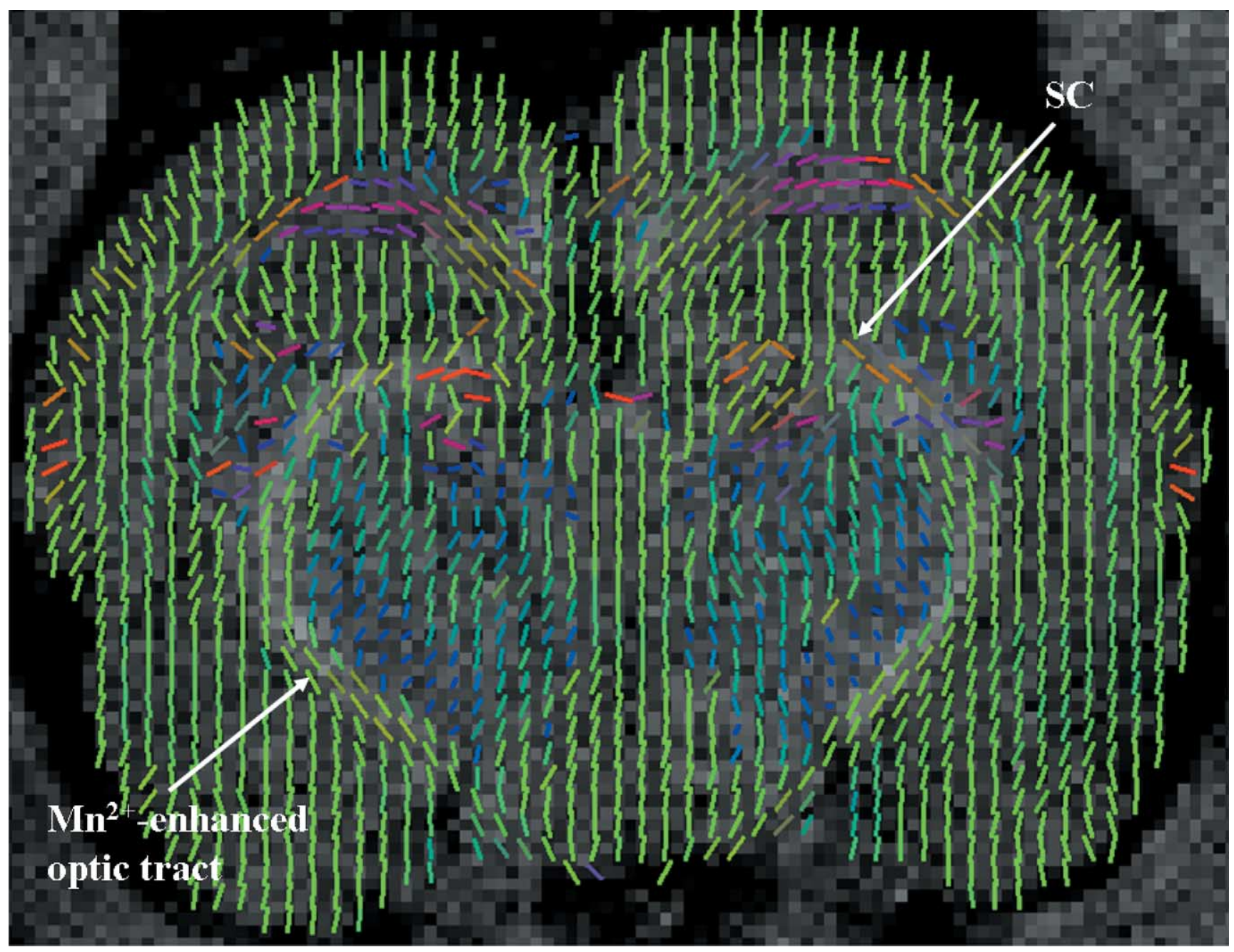

Fig. 5. T1WI of manganese-enhanced optic tracts superimposed with the first eigenvectors of the diffusion tensors. Diffusion tensors were computed from the same DSI data in 515 diffusion-encoding directions. The superimposed image shows that the first eigenvectors of the diffusion tensors, represented by color-coded whiskers, are rather consistent with most of the tract orientation. The consistency seems to break down in regions where the optic tracts merge with the SC, possibly due to crossing fibers from other incoming tracts.

tation of ODF with the same convention used in DTI (Pierpaoli et al., 1996; Jones et al., 1997).

To compare the difference in accuracy between DTI and DSI, we reconstructed diffusion tensor $\mathbf{D}$ from the same spectrum data based on the following relationship

$$
E\left(\mathbf{g}_{i}\right)=\exp \left[-\int_{O}^{\Delta} \mathbf{q}_{i}^{T}(t) \mathbf{D} \mathbf{q}_{i}(t) d t\right],
$$

where $E\left(\mathbf{g}_{i}\right)$ is the echo signal corresponding to a diffusion gradient $\mathbf{g}_{i}$ and $\mathbf{q}_{i}=\gamma \mathbf{g}_{i} \delta / 2 \pi$ (Bloch, 1946; Torrey, 1956; Le Bihan and Basser, 1995; Basser, 2002). The diffusion cross terms induced by the imaging gradients were eliminated after taking geometric mean for the signals that were encoded by diffusion gradients $\mathbf{g}_{i}$ and $-\mathbf{g}_{i}$ (Neeman et al., 1991). Diagonalization of the rank-2 tensor matrix provided the first eigenvector of the diffusion tensor.

Fig. 4. T1WI of manganese-enhanced optic tracts superimposed with ODF orientation. T1WI of a rat brain shows enhancement of the optic tracts from the chiasm to bilateral SC (a). The whisker plot of ODF local maxima superimposed with T1WI, corresponding to an ROI enclosed by a rectangle in (a), shows that the primary orientation of PDF is parallel to the tract orientation (b).

Fig. 8. Whisker plots of the first eigenvectors of the diffusion tensor (a), ODF local maxima (b), and the primary orientation of PDF (c). The three images were superimposed with T1WI of manganese-enhanced optic tracts. In the region of coherent fibers, the orientation shown in (a) through (c) is similar and consistent with the orientation of the enhanced optic tracts. In the region of SC, indicated by rectangles, DTI fails to resolve the fiber orientation due to presence of fiber crossing (a). Orientation of ODF local maxima reveals complex fiber orientation (b), and the primary orientation of PDF correctly defines the optic tract orientation within SC (c). 


\section{Resampling}

We acquired T2WI and DSI of the phantom models under the same imaging conditions. Matrix sizes with $256^{2}$ of T2WI and $32^{2}$ of DSI were resampled for every $8 \times 8$ pixels in T2WI to 1 pixel in DSI. Similarly, we acquired manganese-enhanced TIWI with DSI in one study session with the same slice orientation, thickness, and FOV. Since the matrix sizes for manganese-enhanced T1WI and DSI were $256^{2}$ and $128^{2}$, respectively, we resampled the two images for every $2 \times 2$ pixels in manganese-enhanced MRI corresponding to 1 pixel in DSI.

\section{Deviation angle analysis}

Having aligned T1WI of manganese-enhanced rat optic tracts with DSI, we computed the deviation angle between the primary orientation of PDF projected in the image plane $\mathbf{d}_{1 p}$ and the vector tangent to the optic tract at the same location. Procedures for determining tangential vectors on the manganese-enhanced optic tracts were described previously (Lin et al., 2001). Briefly, we segmented the enhanced tracts with a magnitude threshold, fitted the enhanced pixels with a sixth-order least-square polynomials by the principle of least curvature of fiber tracts (Poupon et al., 2000) and determined the tangential vector of any point on the tract by taking the spatial derivative of the polynomials. The deviation angle was then computed by subtracting the polar angle of $\mathbf{d}_{1 p}$ from the polar angle of the tangential vector. The deviation angle between the first eigenvector of the diffusion tensor and the tangential vector of the optic tract was evaluated in the same way. In ex vivo phantoms, we computed the deviation angle between the primary orientation of PDF and orientation of the crossing capillaries on T2WI. Among all comparisons, we analyzed the variance $\sigma^{2}$ of the deviation angles.

Deviation angle, $\theta$, of the primary orientation of PDF between the spin echo and stimulated echo sequence was computed by

$$
\theta=\arccos \left(\left|\mathbf{v}_{1} \cdot \mathbf{v}_{2}\right|\right) \operatorname{sign}\left(\left(\mathbf{v}_{1} \times \mathbf{v}_{2}\right) \cdot \mathbf{e}_{3}\right),
$$

where $\mathbf{v}_{1}$ and $\mathbf{v}_{2}$ were, respectively, unit vectors along the primary orientation of PDF obtained from the spin echo sequence and stimulated echo sequence, and $\mathbf{e}_{3}$ was the unit vector along the $z$ axis.

\section{Results}

\section{Phantom model}

High-resolution T2WI of both $90^{\circ}$ and $45^{\circ}$ phantoms showed distinct intersecting capillaries orientation by high contrast between water columns and capillary walls (Figs. 2a and 3a). In the same imaging plane, DSI of both $90^{\circ}$ and $45^{\circ}$ phantoms showed stellate patterns of ODF with their main orientation parallel to the orientation of the crossing capillaries (Figs. 2b and 3b). In DTI, the first eigenvectors of the diffusion tensors aligned with noncrossing capillaries. In the regions of crossing fibers, however, the first eigenvectors failed to align with either one of the intersecting capillaries (Figs. 2c and 3c).

\section{Rat model}

Three Wistar rats with a total of six optic tracts were obtained for analysis. Ten hours after manganese injection, T1WI showed good enhancement of the optic tracts from the bilateral retina through the optic chiasm to SC. Fig. 4 shows manganese-enhanced T1WI superimposed on ODF maps. The primary orientation of PDF along the optic tracts was parallel to the orientation of the enhanced tracts. This parallel relationship still maintained in the regions where there were crossing fiber bundles, such as those close to the optic chiasm and those upon entrance to the SC. Although the first eigenvector of the DTI showed equally good correspondence to most segments of the optic tracts, large deviation occurred in the regions of complex fiber crossing (Fig. 5).

\section{Deviation angles}

In the phantom models, the errors of DSI in defining $90^{\circ}$ or $45^{\circ}$ intersecting capillaries were $1.19^{\circ} \pm 4.82^{\circ}$ and $-0.71^{\circ} \pm 4.91^{\circ}$, respectively. Since DTI failed to define either one of the intersecting capillary orientation, its deviation angle could not be analyzed.

For each enhanced optic tract, the deviation angle between the primary orientation of PDF and tangential vector of the tract was analyzed. The total number of pixels analyzed was 134 . The histogram of the deviation angles approximated a normal distribution (mean $\pm \mathrm{SD}$ ) of $-0.41^{\circ}$ $\pm 6.18^{\circ}$. In contrast, the error of DTI in defining fiber orientation was $-1.89^{\circ} \pm 7.7^{\circ}$.

\section{Primary orientation of PDF with $\delta \sim \Delta$ compared with} DSI with $\delta \ll \Delta$

Fig. 6 showed whisker plots of the primary orientation of $\mathrm{PDF}$ in a rat brain obtained from the spin echo sequence (Fig. 6a) and stimulated echo sequence (Fig. 6b). High agreement of the primary orientation can be appreciated in the white matter and most of the cortical gray matter. Large deviation was found in the deep cortical layers, thalamus, hypothalamus, and caudate putamen (Fig. 6c). The total number of pixels analyzed was 4180 . The histogram of the deviation angles approximated a normal distribution (mean $\pm \mathrm{SD}$ ) of $0.87^{\circ} \pm 13.16^{\circ}$ (Fig. 6d). 


\section{Discussion}

By comparing DSI with T2WI of the intersecting capillaries and with T1WI of the manganese-enhanced optic tracts, we quantified the accuracy of DSI in defining crossing fiber orientation. DSI experiments with the condition $\delta \ll \Delta$ were tested in ex vivo phantoms of intersecting capillaries. The deviation angles were $1.19^{\circ} \pm 4.82^{\circ}$ and $-0.71^{\circ} \pm 4.91^{\circ}$ in the $90^{\circ}$ and $45^{\circ}$ intersecting capillaries, respectively. DSI experiments with the condition $\delta \sim \Delta$ were tested in a rat model of manganese-enhanced optic tracts. The deviation angle was $-0.41^{\circ} \pm 6.18^{\circ}$. Comparison of the primary orientation of PDF between a spin echo DSI sequence with $\delta \sim \Delta$ and a stimulated echo DSI sequence with $\delta \ll \Delta$ was tested in a rat brain. The deviation angle was $0.87^{\circ} \pm 13.16^{\circ}$.

\section{Selection of DSI parameters}

DSI describes axonal fiber orientation by mapping 3D PDF of water molecular diffusion within each voxel. The parameters of DSI should be chosen with discretion to ensure adequacy of the resulting PDF to reflect the microstructures. The considerations include choice of diffusion time, $\Delta$, gradient duration $\delta$, sampling interval, $\Delta \mathbf{q}$, and the $\mathbf{q}$ maxima, $\mathbf{q}_{\max }$. Diffusion time should be long enough to ensure that the average diffusion length of water molecules is comparable with the spacing of the restrictive boundaries. In the phantom studies, diffusion time of $250 \mathrm{~ms}$ gave an Einstein length of molecular diffusion of about $(2 D \Delta)^{1 / 2}=$ $33 \mu \mathrm{m}$, more than a half the i.d. of the capillaries. The selection of $\mathbf{q}_{\max }$ should be high enough to ensure that the PDF has sufficient resolution to resolve fiber crossing. Here we used a fixed gradient duration of $6 \mathrm{~ms}$ and the maximal gradient strength of $112 \mathrm{mT} \mathrm{m}^{-1}$. This gave $\mathbf{q}_{\max }=0.028$ $\mu \mathrm{m}^{-1}$ and consequent PDF resolution of $\left(2 \mathbf{q}_{\max }\right)^{-1}=17.6$ $\mu \mathrm{m}$. Knowing that the sampling interval $\Delta \mathbf{q}=\mathbf{q}_{\max } / 5=$ $0.0056 \mu \mathrm{m}^{-1}$, we have FOV of PDF $=(\Delta \mathbf{q})^{-1}=176 \mu \mathrm{m}$. The angular resolution under the current sampling scheme is estimated to be $\arctan (17.6 / 88)=11.3^{\circ}$.

In the rat model, we used a spin echo pulsed gradient sequence with $\Delta=25 \mathrm{~ms}$, which gave an Einstein length of molecular diffusion of about $10.5 \mu \mathrm{m}$, close to an average axonal fiber diameter of about $10 \mu \mathrm{m}$. The gradient duration of $17 \mathrm{~ms}$ and the maximal gradient strength of $180 \mathrm{mT} \mathrm{m}^{-1}$ were used for the rat experiment. This gave $\mathbf{q}_{\max }=0.13$ $\mu \mathrm{m}^{-1}$. It follows that PDF resolution of $\left(2 \mathbf{q}_{\max }\right)^{-1}=3.84$ $\mu \mathrm{m}$ and FOV of PDF $=10 \times 3.84=38.4 \mu \mathrm{m}$. Again, angular resolution is $\arctan (3.84 / 19.2)=11.3^{\circ}$, the same as that in the phantom experiment. Similarly, in the comparison of the primary orientation of PDF the same considerations were used to choose the appropriate $\Delta, \Delta \mathbf{q}$, and $\mathbf{q}_{\max }$ for both spin echo DSI and stimulated echo DSI.

\section{Ex vivo phantom model}

In this study, we used water-filled plastic capillaries with the i.d. $=50 \mu \mathrm{m}$ and o.d. $=350 \mu \mathrm{m}$ to simulate the biological axonal fiber crossing. Although an i.d. of $50 \mu \mathrm{m}$ was larger than the average scale of axonal fiber diameter, about $10 \mu \mathrm{m}$, boundary effects on restrictive diffusion still existed despite the diffusion signal being weak. Indeed, the same type of capillaries was adopted by von dem Hagen and Henkelman (2002) to simulate the displacement distribution of the diffusion tensor. Another concern might be raised regarding to the accuracy of DTI derived from the existing DSI data since most of the $b$ values in the present DSI data were higher than those used in standard DTI. Fig. 7 shows the results of DSI and DTI in defining capillaries of coherent orientation. As compared with high-resolution T2WI of the capillary phantoms, the accuracy of DSI and DTI was similar, $0.22^{\circ} \pm 4.56^{\circ}$ and $0.68^{\circ} \pm 4.12^{\circ}$, respectively. High accuracy of DTI in defining coherent capillaries ensured that the accuracy of DTI was not compromised by the current data acquisition scheme. Thus, it is legitimate to compare the accuracy of DSI and DTI in crossing capillaries.

\section{Error estimate}

Our results showed that under the current data acquisition scheme, the error of DSI in defining fiber orientation was $-0.41^{\circ} \pm 6.18^{\circ}$. To compare DTI and DSI under the same imaging conditions, DTI derived from the same data of DSI was analyzed. The error of DTI in defining optic tract was $-1.89^{\circ} \pm 7.7^{\circ}$. Careful inspection of the DSI revealed that there were crossing fibers in the regions close to the SC and the optic chiasm (Fig. 8). In these regions, the first eigenvectors of the diffusion tensors deviated from the tract orientation substantially. If these pixels were excluded, the error of DTI reduced to $-0.9^{\circ} \pm 6.38^{\circ}$, comparable to that of DSI. This result indicated that DTI had the same accuracy as DSI for coherent fibers, but the accuracy of DTI deteriorated in the presence of fiber crossing.

Our previous report showed that, excluding the effect of MR noise, the deviation between standard DTI acquisition scheme and manganese-enhanced optic tracts had an error of $7.82^{\circ}$ (Lin et al., 2001). This error can be decomposed into two parts. One represented an error from the fitting procedure of the enhanced tracts $\left(5.11^{\circ}\right)$. The other represented an error not related to MR noise but arising from bias inherent to the DTI method $\left(5.92^{\circ}\right)$ such as eddy currents, diffusion cross-terms, choice of diffusion-encoding schemes, and intravoxel incoherent fibers. Under the current acquisition scheme, we found that the deviation between DTI and manganese-enhanced optic tracts was $7.7^{\circ}$, comparable to the error of $7.82^{\circ}$. This means the error of DTI can be explained by the error from the fitting procedure and the error inherent in the DTI method. The effect of MR 

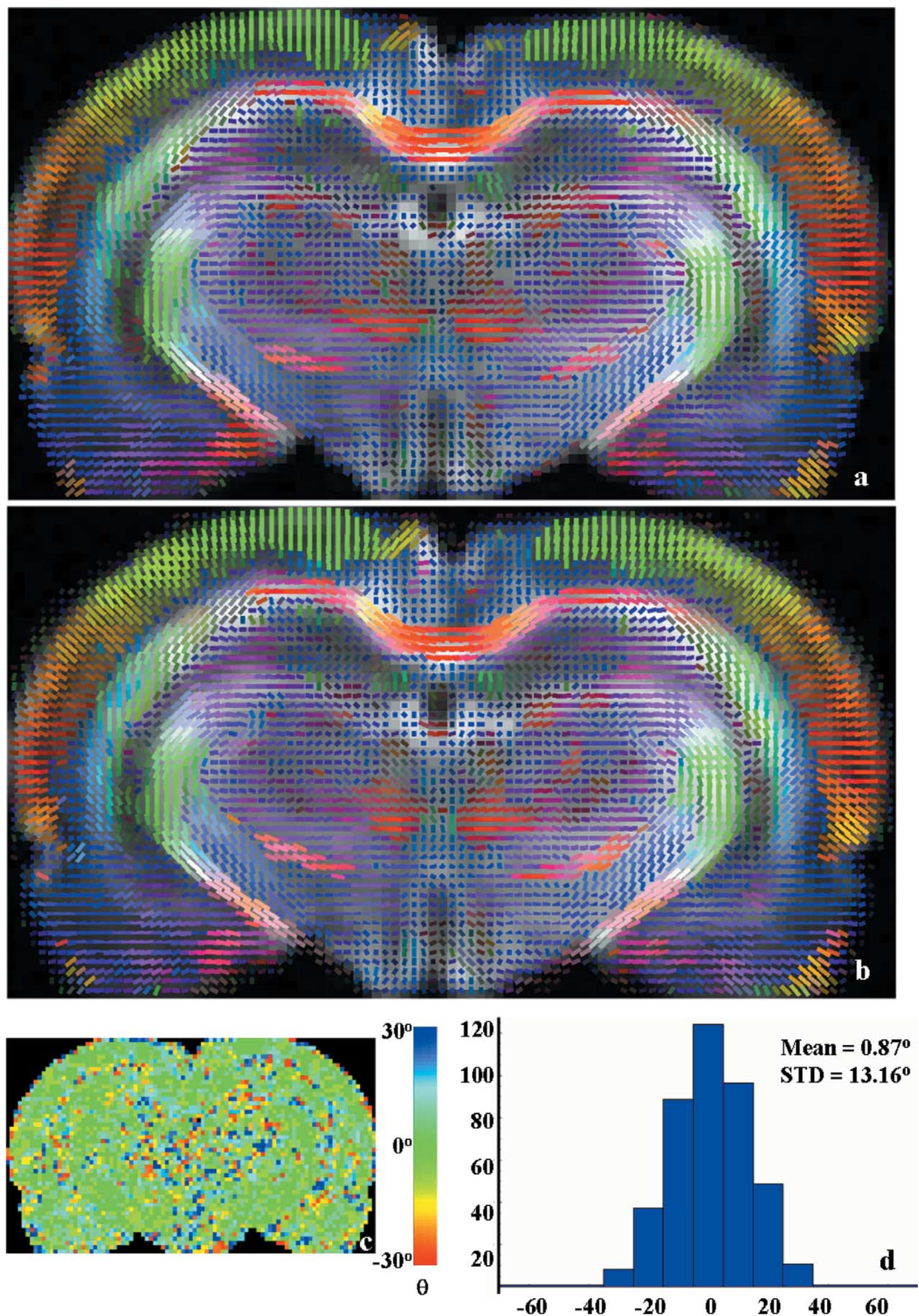

Fig. 6. Whisker plots of the primary orientation of PDF of a rat brain obtained from a spin echo DSI sequence (a), a stimulated echo DSI sequence (b). The PDF orientation at the same location is very similar in two images. Deviation angle map shows large deviation in deep cortical layers, thalamus, hypothalamus, and caudate putamen, corresponding to complex fiber crossing or prominent aggregation of the neurons (c). The histogram of the deviation angles approximates a normal distribution with a mean $\pm \mathrm{SD}$ of $0.87^{\circ} \pm 13.16^{\circ}$. 

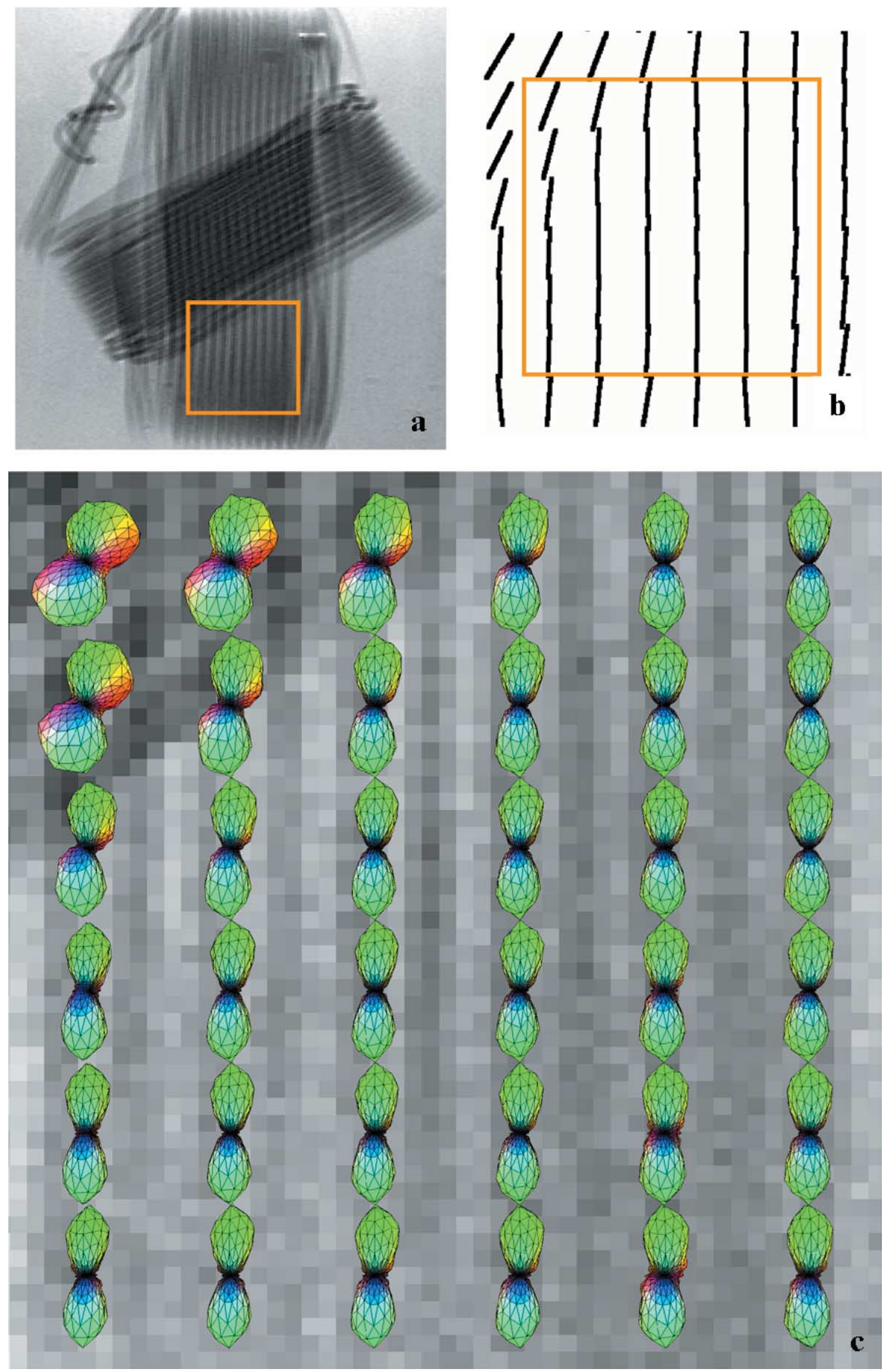

Fig. 7. The phantom of coherent capillaries. Compared with high-resolution T2WI in (a), both first eigenvector of the diffusion tensor (b) and ODF (c) define the capillary orientation correctly. The rectangles enclose the same ROI as that in (c). 
noise is negligible. It should be noted that the error of $7.82^{\circ}$ was computed from the entire optic tracts, including coherent and incoherent portions. As described in the previous paragraph, the errors of DSI and DTI for coherent fibers were $6.18^{\circ}$ and $6.38^{\circ}$, respectively. Acknowledging this, we estimated the error from the intravoxel incoherent fibers to be $\left(7.82^{2}-6.38^{2}\right)^{1 / 2}=4.52^{\circ}$. Again, the observed errors in coherent fibers suggested that under the current acquisition scheme the errors of DSI and DTI in defining tract orientation are close to the noise limit.

\section{Effects of finite gradient pulse widths on fiber orientation}

Since the essential requirement $\delta \ll \Delta$ for the Fourier relation between the echo signal and the PDF is never attainable in practice, effects of finite gradient pulse widths, $\delta$, on echo signal, $E(\mathbf{q})$, with respect to microstructural dimensions, denoting $\mathbf{a}$, have been studied by many groups (Blees, 1994; Coy and Callaghan, 1994; Mitra and Halperin, 1995; Caprihan et al., 1996; Callaghan, 1997; Mair et al., 2002). Blees and Caprihan et al. found that as $\delta$ increases, the local maxima and minima of $E(\mathbf{q})$ showed a shift to right in the qa axis, indicating underestimate of a. Further, as a decreases the underestimate becomes more prominent. These effects may cause problems in determining the actual dimensions of the microstructures, they might not be as problematic in defining the orientation of the local maxima from the apparent 3D PDF. In fact, the effects of finite gradient pulse widths described above do not alter the order of local maxima, but rather enhance the contrast between local maxima in different directions.

The deviation angle $\theta$ of the primary orientation of PDF between the spin echo and stimulated echo sequence was $0.87^{\circ} \pm 13.16^{\circ}$ throughout the whole imaged brain. The total error of this result comprises three parts, the error related to MR noise, a bias inherent to the DSI method, and a bias between these two sequences. Taking the error of DSI in the rat model into consideration, we estimate that the bias between the two sequences is approximately $\left(13.16^{2}-2 \times\right.$ $\left.6.18^{2}\right)^{1 / 2} \sim 10^{\circ}$. Another supportive evidence is from our rat model results, showing that the primary orientation of the PDF follows the orientation of optic nerves accurately, not significantly affected by the finite gradient pulse width.

\section{Limitations}

In the in vivo study, we found that the primary orientation of PDF accurately defined optic tract orientation even in the presence of crossing from the other tracts. Though in the ex vivo phantoms both primary and secondary orientation of PDF accurately aligned with the crossing capillaries, it does not imply that in complex fiber geometry of the brain, another orientation of the PDF is as accurate as the primary one. Further study is required to validate the accuracy of PDF orientation of higher orders. The diffusion- sensitive gradients used in both ex vivo and in vivo studies exceed $100 \mathrm{mT} \mathrm{m}^{-1}$, larger than $40 \mathrm{mT} \mathrm{m}^{-1}$ used in the clinical scanner. To have $b_{\max }=13,000 \mathrm{~s} \mathrm{~mm}^{-2}$ attained in a clinical scanner, approximately $55 \mathrm{~ms}$ is required for $\delta$ and $\Delta$. According to Blees' analysis, the underestimate of dimensions would be more prominent than that in our study where $\delta \sim \Delta=20 \mathrm{~ms}$ (Blees, 1994). As discussed previously, an underestimate of dimensions does not necessarily affect the orientation of 3D PDF. However, further study is needed to understand the effect of change in sensitivity of underestimate on the PDF orientation.

\section{Conclusion}

Using capillary phantoms and a rat model of manganeseenhanced optic tracts, we have quantified the accuracy of DSI in defining crossing fiber orientation. In experiments of the phantom models where $\delta \ll \Delta$ was met, DSI was able to define orientation of crossing capillaries with an error of less than $5^{\circ}$. In experiments of the rat models where a finite gradient pulse width was used, the accuracy of DSI in defining crossing fiber orientation was about $6^{\circ}$, close to the noise limit. In the comparison between short and long pulsed gradient sequences, the bias was approximately $10^{\circ}$. Therefore, we conclude that DSI can resolve crossing fibers accurately. The effect of finite gradient pulse widths on the primary orientation is not critical.

\section{Acknowledgments}

The authors are indebted to Dr. Chen-Tung Yen and Dr. Keng-Chen Liang for their helpful advice to this study. This study is supported in part by the National Health Research Institutes Grant NHRI-EX90-9018EP, the Program for Promoting Academic Excellence of Universities A-91-NFA01-2-4, and the National Institute of Health Grant MH64044.

\section{References}

Assaf, Y., Cohen, Y., 1999. Structural information in neuronal tissue as revealed by q-space diffusion NMR spectroscopy of metabolites in bovine optic nerve. NMR Biomed. 12 (6), 335-344.

Assaf, Y., Mayk, A., Cohen, Y., 2000. Displacement imaging of spinal cord using q-space diffusion-weighted MRI. Magn. Reson. Med. 44 (5), 713-722.

Balinov, B., Jonsson, B., Linse, P., Soderman, O., 1993. The NMR selfdiffusion method applied to restricted diffusion: simulation of echo attenuation from molecules in spheres and between planes. J. Magn. Reson. A 104, 17-25.

Basser, P.J., 2002. Relationships between diffusion tensor and q-space MRI. Magn. Reson. Med. 47 (2), 392-397.

Basser, P.J., Mattiello, J., LeBihan, D., 1994. MR diffusion tensor spectroscopy and imaging. Biophys. J. 66 (1), 259-267. 
Basser, P.J., Pajevic, S., Pierpaoli, C., Duda, J., Aldroubi, A., 2000. In vivo fiber tractography using DT-MRI data. Magn. Reson. Med. 44 (4), 625-632.

Blees, M.H., 1994. The effect of finite duration of gradient pulses on the pulsed-field-gradient NMR method for studying restricted diffusion. J. Magn. Reson. A 109, 203-209.

Bloch, F., 1946. Nuclear induction. Phys. Rev. 70, 460-474.

Callaghan, P.T., 1991. Principles of Nuclear Magnetic Resonance Microscopy. Clarendon Press, Oxford.

Callaghan, P.T., 1996. NMR imaging, NMR diffraction and applications of pulsed gradient spin echoes in porous media. Magn. Reson. Imaging 14 (7-8), 701-709.

Callaghan, P.T., 1997. A simple matrix formalism for spin echo analysis of restricted diffusion under generalized gradient waveforms. J. Magn Reson. 129, 74-84.

Callaghan, P.T., Coy, A., MacGowan, D., Packer, K.J., Zelaya, F.O., 1991. Diffraction-like effects in NMR diffusion studies of fluids in porous solids. Nature 351, 467-469.

Callaghan, P.T., Eccles, C.D., Xia, Y., 1988. NMR microscopy displacements: k-space and q-space imaging. J. Phys. E 21, 820.

Caprihan, A., Wang, L.Z., Eiichi, F., 1996. A multiple-narrow-pulse approximation for restricted diffusion in a time-varying field gradient. J. Magn. Reson. A 118, 94-102.

Conturo, T.E., Lori, N.F., Cull, T.S., Akbudak, E., Snyder, A.Z., Shimony, J.S., McKinstry, R.C., Burton, H., Raichle, M.E., 1999. Tracking neuronal fiber pathways in the living human brain. Proc. Natl. Acad. Sci. USA 96 18, 10422-10427.

Cory, D.G., Garroway, A.N., 1990. Measurement of translational displacement probabilities by NMR: an indicator of compartmentation. Magn Reson. Med. 14 (3), 435-444.

Coy, A., Callaghan, P.T., 1994. Pulsed gradient spin echo nuclear magnetic resonance for molecules diffusing between partially reflecting rectangular barriers. J. Chem. Phys. 101, 4599-4609.

Frank, L.R., 2001. Anisotropy in high angular resolution diffusionweighted MRI. Magn. Reson. Med. 45 (6), 935-939.

Frank, L.R., 2002. Characterization of anisotropy in high angular resolution diffusion-weighted MRI. Magn. Reson. Med. 47 (6), 1083-1099.

Gossl, C., Fahrmeir, L., Putz, B., Auer, L.M., Auer, D.P., 2002. Fiber tracking from DTI using linear state space models: detectability of the pyramidal tract. NeuroImage 16 (2), 378-388.

Grannell, P.K., Mansfield, P., 1975. Microscopy in vivo by nuclear magnetic resonance. Phys. Med. Biol. 20 (3), 477-482.

Hills, B.P., Wright, K.M., Snaar, J.E., 1996. Dynamic NMR q-space studies of microstructure with the multigrade CPMG sequence. Magn. Reson. Imag. 14 (3), 305-318.

Jones, D.K., Williams, S.C., Horsfield, M.A., 1997. Full representation of white-matter fibre direction on one map via diffusion tensor analysis. Proc. Int. Soc. Magn. Reson. Med. ISMRM, California, p. 1743.

King, M.D., Houseman, J., Roussel, S.A., van Bruggen, N., Williams, S.R., Gadian, D.G., 1994. q-Space imaging of the brain. Magn. Reson. Med. 32 (6), 707-713

Kuchel, P.W., Durrant, C.J., Chapman, B.E., Jarrett, P.S., Regan, D.G., 2000. Evidence of red cell alignment in the magnetic field of an NMR spectrometer based on the diffusion tensor of water. J. Magn. Reson. 145 (2), 291-301.
Le Bihan, D., Basser, P.J., 1995. Molecular diffusion and nuclear magnetic resonance. in: Le Bihan, D (Ed.), Diffusion and Perfusion Magnetic Resonance Imaging: Applications to Functional MRI. Raven Press, New York, pp. 5-17.

Lin, C.P., Tseng, W.Y., Cheng, H.C., Chen, J.H., 2001. Validation of diffusion tensor magnetic resonance axonal fiber imaging with registered manganese-enhanced optic tracts. NeuroImage 14 5, 1035-1047.

Mair, R.W., Sen, P.N., Hurlimann, M.D., Patz, S., Cory, D.G., Walsworth, S.L., 2002. The narrow pulse approximation and long length scale determination in xenon gas diffusion NMR studies of model porous media. J. Magn. Reson. 156, 202-212.

Mansfield, P., Grannell, P.K., 1973. NMR ‘diffraction' in solids. J. Phys. C6, L422-L426.

Mitra, P.P., Halperin, B.J., 1995. Effects of finite gradient-pulse widths in pulsed-field-gradient diffusion measurements. J. Magn. Reson. 113, 94-101.

Mori, S., Crain, B.J., Chacko, V.P., van Ziji, P.C., 1999. Three-dimensional tracking of axonal projections in the brain by magnetic resonance imaging. Ann. Neurol. 45 (2), 265-269.

Neeman, M., Freyer, J.P., Sillerud, L.O., 1991. A simple method for obtaining cross-term-free images for diffusion anisotropy studies in NMR microimaging. Magn. Reson. Med. 21 (1), 138-143.

Parker, G.J., Stephan, K.E., Barker, G.J., Rowe, J.B., MacManus, D.G., Wheeler-Kingshott, C.A., Ciccarelli, O., Passingham, R.E., Spinks, R.L., Lemon, R.N., Turner, R., 2002. Initial demonstration of in vivo tracing of axonal projections in the macaque brain and comparison with the human brain using diffusion tensor imaging and fast marching tractography. NeuroImage 15 (4), 797-809.

Pierpaoli, C., Jezzard, P., Basser, P.J., Barnett, A., Di Chiro, G., 1996. Diffusion tensor MR imaging of the human brain. Radiology 2013 , $637-648$

Poupon, C., Clark, C.A., Frouin, V., Regis, J., Bloch, I., Le Bihan, D., Mangin, J., 2000. Regularization of diffusion-based direction maps for the tracking of brain white matter fascicles. NeuroImage 12 (2), $184-$ 195.

Stejskal, E.O., 1965. Use of spin echoes in a pulsed magnetic-field gradient to study restricted diffusion and flow. J. Chem. Phys. 43, 3597-3603.

Stieltjes, B., Kaufmann, W.E., van Zijl, P.C., Fredericksen, K., Pearlson, G.D., Solaiyappan, M., Mori, S., 2001. Diffusion tensor imaging and axonal tracking in the human brainstem. NeuroImage 14 (3), 723-735.

Torrey, H.C., 1956. Bloch equations with diffusion terms. J. Chem. Phys. 104 (3), 563-565

Tuch, D.S., Weisskoff, R.M., Belliveau, J.W., Wedeen, V.J., 1999. High angular resolution diffusion imaging of the human brain. Proc. Int. Soc. Magn. Reson. Med. ISMRM, California, p. 321.

von dem Hagen, E.A., Henkelman, R.M., 2002. Orientational diffusion reflects fiber structure within a voxel. Magn. Reson. Med. 48 (3), 454-459.

Wedeen, V.J., Reese, T.G., Tuch, D.S., Weigel, M.R., Dou, J.-G., Weiskoff, R.M., Chessler, D.,2000 Mapping fiber orientation spectra in cerebral white matter with Fourier-transform diffusion MRI. Proc. Int. Soc. Magn. Reson. Med. ISMRM, California, p. 82.

Wiegell, M.R., Larsson, H.B., Wedeen, V.J., 2000. Fiber crossing in human brain depicted with diffusion tensor MR imaging. Radiology 217 (3), 897-903 